\title{
Allele specific expression analysis identifies regulatory variation associated with stress-related genes in the Mexican highland maize landrace Palomero Toluqueño
}

\author{
M. Rocío Aguilar-Rangel ${ }^{1,2}$ ， Ricardo A. Chávez Montes ${ }^{1,3}$ ， Eric González-Segovia ${ }^{1}$ ， Jeffrey Ross-Ibarra ${ }^{4}$, June \\ K Simpson ${ }^{2}$, Ruairidh J H Sawers ${ }^{\text {Corresp. } 1}$ \\ 1 Unidad de Genómica Avanzada (LANGEBIO), Centro de Investigación y de Estudios Avanzados del Instituto Politécnico Nacional, Irapuato, Guanajuato, \\ Mexico \\ 2 Departamento de Ingeniería Genética, Centro de Investigación y de Estudios Avanzados del Instituto Politécnico Nacional (CINVESTAV-IPN), Irapuato, \\ Guanajuato, Mexico \\ 3 ABACUS: Laboratorio de Matemáticas Aplicadas y Cómputo de Alto Rendimiento del Departamento de Matemáticas, Centro de Investigación y de \\ Estudios Avanzados del Instituto Politécnico Nacional (CINVESTAV-IPN), Ocoyoacac, Estado de México, Mexico \\ 4 Department of Plant Sciences, Center for Population Biology and Genome Center, University of California, Davis, Davis, CA, United States \\ Corresponding Author: Ruairidh J H Sawers \\ Email address: rusawers@cinvestav.mx
}

Background. Gene regulatory variation has been proposed to play an important role in the adaptation of plants to environmental stress. In the central highlands of Mexico, farmer selection has generated a unique group of maize landraces adapted the challenges of the highland niche. In this study, gene expression in Mexican highland maize and a reference maize breeding line were compared to identify evidence of regulatory variation in stress-related genes. It was hypothesised that local adaptation in Mexican highland maize would be associated with a transcriptional signature observable even under benign conditions

Methods. Allele specific expression analysis was performed using the seedling-leaf transcriptome of an $\mathrm{F}_{1}$ individual generated from the cross between the highland adapted Mexican landrace Palomero Toluqueño and the reference line B73, grown under benign conditions. Results were compared with a published dataset describing the transcriptional response of B73 seedlings to cold, heat, salt and UV treatments

Results. A total of 2386 genes were identified to show allele specific expression. Of these, 277 showed an expression difference between Palomero Toluqueño and B73 alleles under benign conditions that anticipated the response of B73 cold, heat, salt and/or UV treatments, and, as such, were considered to display a prior stress response. Prior stress response candidates included genes associated with plant hormone signaling and a number of transcription factors. Construction of a gene co-expression network revealed further signaling and stress-related genes to be among the potential targets of the transcription factors candidates.

Discussion. Prior activation of responses may represent the best strategy when stresses are severe but predictable. Expression differences observed here between Palomero Toluqueño and B73 alleles indicate the presence of cis-acting regulatory variation linked to stress-related genes in Palomero Toluqueño. Considered alongside gene annotation and population data, allele specific expression analysis of plants grown under benign conditions provides an attractive strategy to identify functional variation potentially linked to local adaptation. 
Allele specific expression analysis identifies regulatory variation associated with stress-related genes in the Mexican highland maize landrace Palomero Toluqueño

M. Rocío Aguilar-Rangel ${ }^{1,2} \psi$, Ricardo A. Chávez Montes ${ }^{1,3} \psi$, Eric Gonzalez-Segovia ${ }^{1}$, Jeffrey Ross-Ibarra $^{4}$, June K. Simpson ${ }^{2}$, Ruairidh J. H. Sawers ${ }^{1}$

${ }^{1}$ Unidad de Genómica Avanzada (LANGEBIO), Centro de Investigación y de Estudios Avanzados del Instituto Politécnico Nacional, Km. 9.6 Libramiento Norte, Carretera IrapuatoLeón, CP 36821 Irapuato, Guanajuato, México.

${ }^{2}$ Departamento de Ingeniería Genética, Centro de Investigación y de Estudios Avanzados del Instituto Politécnico Nacional (CINVESTAV-IPN), Km. 9.6 Libramiento Norte, Carretera Irapuato-León, CP 36821 Irapuato, Guanajuato, México.

${ }^{3}$ ABACUS: Laboratorio de Matemáticas Aplicadas y Cómputo de Alto Rendimiento del Departamento de Matemáticas, Centro de Investigación y de Estudios Avanzados del Instituto Politécnico Nacional (CINVESTAV-IPN), Carretera México-Toluca Km. 38.5, Ocoyoacac, Estado de México, México.

${ }^{4}$ Department of Plant Sciences, Center for Population Biology, and Genome Center, University of California, Davis, CA, 95616, USA.

$\psi$ These authors contributed equally to this work

Corresponding Author:

Ruairidh Sawers ${ }^{1}$

e-mail address: rusawers@cinvestav.mx 


\section{ABSTRACT}

2 Background. Gene regulatory variation has been proposed to play an important role in the

3 adaptation of plants to environmental stress. In the central highlands of Mexico, farmer selection

4 has generated a unique group of maize landraces adapted the challenges of the highland niche. In

5 this study, gene expression in Mexican highland maize and a reference maize breeding line were

6 compared to identify evidence of regulatory variation in stress-related genes. It was hypothesised

7 that local adaptation in Mexican highland maize would be associated with a transcriptional

8 signature observable even under benign conditions.

9 Methods. Allele specific expression analysis was performed using the seedling-leaf

transcriptome of an $F_{1}$ individual generated from the cross between the highland adapted

11 Mexican landrace Palomero Toluqueño and the reference line B73, grown under benign

12 conditions. Results were compared with a published dataset describing the transcriptional

13 response of B73 seedlings to cold, heat, salt and UV treatments.

14 Results. A total of 2386 genes were identified to show allele specific expression. Of these, 277

15 showed an expression difference between Palomero Toluqueño and B73 alleles under benign

16 conditions that anticipated the response of B73 cold, heat, salt and/or UV treatments, and, as

17 such, were considered to display a prior stress response. Prior stress response candidates included

18 genes associated with plant hormone signaling and a number of transcription factors.

19 Construction of a gene co-expression network revealed further signaling and stress-related genes

20 to be among the potential targets of the transcription factors candidates.

21 Discussion. Prior activation of responses may represent the best strategy when stresses are severe

22 but predictable. Expression differences observed here between Palomero Toluqueño and B73

23 alleles indicate the presence of cis-acting regulatory variation linked to stress-related genes in 
24 Palomero Toluqueño. Considered alongside gene annotation and population data, allele specific 25 expression analysis of plants grown under benign conditions provides an attractive strategy to 26 identify functional variation potentially linked to local adaptation.

27

28 


\section{INTRODUCTION}

30 Extensive study across different plant species has identified a range of transcriptional responses

31 to abiotic stresses. Although basic responses are typically conserved, variation in the regulation

32 of stress-responsive genes has been observed among individuals and varieties, potentially playing

33 an important role in adaptation to stressful environments (Hannah et al., 2006); (Swanson-

34 Wagner et al., 2012); (Rengel et al., 2012); (Lasky et al., 2014). From an agronomic perspective,

35 biotechnological approaches to enhance crop stress tolerance to abiotic stress often aim to

36 manipulate gene expression rather than engineer protein sequences (e.g. (Kamthan et al., 2016)).

37 Similarly, efforts to identify suitable material for breeding towards similar goals have drawn on

38 natural cis-acting regulatory variation acting on stress-responsive gene expression (e.g. (Mao et

39 al., 2015)). As such efforts are intensified in the face of mounting concern regarding the impact

40 of climate change on crop productivity, there is ever greater interest in the genetic basis of

41 variation in stress-responses (Des Marais, Hernandez \& Juenger, 2013).

42 Crop landrace varieties represent an invaluable genetic resource. Collectively, the range

43 of environments exploited by landraces typically exceeds that of improved varieties, and many

44 landraces are adapted to conditions that would be considered stressful in conventional agriculture

45 (Ruiz Corral et al., 2008) (Romero Navarro et al., 2017). Nonetheless, although landraces

46 represent a compelling source for enhancing abiotic stress tolerance in breeding programs, the

47 task of identifying useful genetic variants and transferring them to breeding material is far from

48 trivial (Sood et al., 2014). In addition to the complication of working with often heterogenous

49 landrace germplasm, reproducing stress conditions for evaluation is costly and difficult.

50 Furthermore, stress is not well reflected by a single experimental treatment, but rather represents

51 a continuous environmental range defined by interacting variables acting over the lifetime of the 
52 plant. Large-scale phenomics efforts are an attempt to implement the factorial designs required to 53 capture such complexity (Houle, Govindaraju \& Omholt, 2010); (Furbank \& Tester, 2011), but

54 they require a substantial investment in infrastructure that may not be feasible in many research contexts. A number of approaches aim to leave aside such difficulties, and to identify candidate genes directly from genomic data through the incorporation of environmental variables into population genetic and genome wide association studies (Coop et al., 2010);(Laskey et al., 2015). Here, as a further alternative, transcriptome data is explored for signatures of an enhanced stress response hardwired in locally adapted material, and evident under benign conditions.

Stress responses are considered to be an adaptation to an unpredictable, often suboptimal environment. Under benign conditions, however, activation of these same pathways, by exogenous application of plant hormones or mutation of genes involved in signaling pathways, is associated with growth retardation (Staswick et al., 1992);(Hu et al, 1996);(Bowling et al., 1997);(Ellis \& Turner, 2001), indicating both their potential cost to the plant, and the benefit of maintaining tight regulation. Nonetheless, when conditions are adverse, but predictably so, it may be advantageous to anticipate activation of stress pathways and avoid the delay between stimulus and response inherent in plasticity (Levins, 1968); (von Heckel, Stephan \& Hutter, 2016). In cultivated systems, non-adapted varieties can benefit from mild priming stress treatments that activate protective mechanisms and prepare the plants for future more severe environmental challenges (van Hulten et al., 2006);(Hilker et al., 2016). In practice, however, the

71 first exposure to a stress may be severe, placing the unprepared organism at risk. Here, the

72 hypothesis is addressed that anticipation of stress responses is a hallmark of local adaptation to

73 marginal environments, presenting an opportunity to identify genetic variation related to

74 enhanced stress tolerance that is expressed under benign conditions. 

a powerful approach to identify the molecular mechanisms underlying tolerance variation (e.g.

77 (Hayano-Kanashiro et al., 2009); (von Heckel, Stephan \& Hutter, 2016). The number of

78 differentially accumulating transcripts, however, may be large, and the data reflect both cisacting and trans-acting regulatory variation. Critically, per se comparison of varieties has little power to characterize the genetic architecture of stress tolerance or to identify causative genetic variation. In addition, when material is diverse, phenological differences can make it difficult to devise an appropriate sampling strategy. With the development of sequencing based methods to study the transcriptome, it is possible to make use of natural sequence variation to quantify allele specific expression (ASE) in $\mathrm{F}_{1}$ hybrid individuals generated from the cross of two different lines of interest (Springer \& Stupar, 2007b); (Springer \& Stupar, 2007a); (Zhang \& Borevitz, 2009);

86 (Lemmon et al., 2014). Characterization of ASE in $\mathrm{F}_{1}$ material avoids the problems of

87 comparing parents that may be very different in growth and development by evaluating both

88 alleles within the same cellular environment, directly revealing cis-acting genetic variation for

89 transcript accumulation (Springer \& Stupar, 2007b); (Lemmon et al., 2014); (Waters et al., 90 2017).

In this study, a transcriptome dataset was examined for evidence of cis-regulatory

92 variation linked to stress-associated genes in Palomero Toluqueño (PT), a maize landrace 93 originating from the highlands of Central Mexico (Prasanna, 2012); (Perales \& Golicher, 2014).

94 The Mexican highland environment exposes maize plants to a number of abiotic stresses:

95 bringing plants to maturity under low-temperatures necessitates planting early in the year, 96 exposing seedlings to late frosts and water deficit before onset of the annual rains; throughout the 97 growing season, low-temperature, high-levels of UV radiation and hail storms pose further 
98 challenges (Eagles \& Lothrop, 1994); (Lafitte \& Edmeades, 1997); (Jiang et al., 1999); (Mercer,

99 Martínez-Vásquez \& Perales, 2008); (Ruiz Corral et al., 2008). The unique group of Mexican

100 highland maize landraces, including PT, has been shown previously to be superior in the

101 highland niche to maize originating from temperate, mid-altitude tropical or lowland tropical

102 regions with respect to seedling emergence, photosynthetic efficiency, and tolerance to frost,

103 cold, drought and hail (Eagles \& Lothrop, 1994); (Mercer, Martínez-Vásquez \& Perales, 2008).

104 To identify evidence of regulatory variation that might underlie adaptation to these conditions, an

$105 \mathrm{~F}_{1}$ was generated between PT and the midwest-adapted maize reference line B73, and the leaf

106 transcriptome analyzed under benign greenhouse conditions to detect ASE. Results of the

107 analysis were compared with a published study in which B73 seedlings were exposed to cold,

108 heat, salt and UV stress treatments (Makarevitch et al., 2015). A total of 277 genes were

109 identified showing a pattern of ASE under benign conditions that mirrored the response of the

110 same gene under stress in B73, hereafter referred to as prior stress response (PSR). The PSR

111 candidate set included transcription factors and genes associated with plant hormone signalling, a

112 number of which are discussed in more detail and presented as candidates for future functional

113 analysis.

115 MATERIALS AND METHODS

116 Plant material, RNA preparation, and sequencing

117 Seed of the Mexican highland landrace Palomero Toluqueño accession Mexi5 was obtained from

118 the International Maize and Wheat Improvement Center (CIMMYT; stock GID 244857). The

119 original collection was made near the city of Toluca, in Mexico state (19.286184 N, -99.570871

$120 \mathrm{~W}$ ), at an elevation of 2597 masl. An $\mathrm{F}_{1}$ hybrid stock was generated from the cross between the 
121 inbred line B73 and PT grown under standard greenhouse conditions $\left(27^{\circ} \mathrm{C}\right.$ day, $24^{\circ} \mathrm{C}$ nights; 15

122 hour days, 9 hour nights; 30\% humidity. Ames, Iowa) and total RNA was extracted from leaf

123 tissue of a single, 14 day-old seedling using the Qiagen RNeasy Plant Mini Kit (cat ID 74904)

124 according to the manufacturer's protocol. RNA integrity was assessed by spectrophotometry and

125 agarose gel electrophoresis. Library preparation was performed using the Illumina protocol as

126 outlined in the TruSeq RNA Sample Preparation Guide (15008136 A, November 2010) and

127 paired-end sequencing was carried out on the Illumina HiSeq 2000 platform. Raw data is

128 available in the NCBI (www.ncbi.nlm.nih.gov) Sequence Read Archive under accession

129 SRP011579. Plant growth conditions in the Makarevitch study (Makarevitch et al., 2015) were

130 described previously in the published report. Briefly, whole above ground tissue was collected

131 for 14 day old seedlings. For cold stress, seedlings were incubated at $5^{\circ} \mathrm{C}$ for 16 hours. For heat

132 stress, seedlings were incubated at $50^{\circ} \mathrm{C}$ for 4 hours. For high salt stress, plants were watered

133 with $300 \mathrm{mM} \mathrm{NaCl} 20$ hours prior to tissue collection. For UV stress, plants were irradiated using

134 UV-B lamps for 2 hours prior to tissue collection.

136 Allele Specific Expression (ASE) analysis

137 Allele specific expression (ASE) analysis was based on the method of Lemmon and collaborators

138 (Lemmon et al., 2014) and the detailed pipeline is presented as Supplementary Data 1 [pipeline].

139 A set of 39475 B73 transcripts was generated by selecting the longest predicted transcript for

140 each gene annotated in the AGPv3.22 B73 reference genome

141 (ftp://ftp.ensemblgenomes.org/pub/release-22/). Six transcripts whose sequences consisted of

142 only, or mostly, undefined (N) bases were removed (GRMZM2G031216_T01,

143 GRMZM2G179334_T01, GRMZM2G307432_T01, GRMZM2G316264_T01, 
144 GRMZM2G406088_T01 and GRMZM2G700875_T01), resulting in a set of 39469 sequences. A

145 total of $151,168,196$ paired-end reads from the $\mathrm{B} 73 \times \mathrm{XT} \mathrm{F}_{1}$ transcriptome were trimmed using

146 Trimmomatic (Bolger, Lohse \& Usadel, 2014) and aligned using bwa mem (Li, 2013) to the set

147 of B73 transcripts. The resulting alignment was processed using samtools, bcftools and vcfutils

148 (Li et al., 2009); (Li, 2011a); (Li, 2011a,b) to identify polymorphisms. We then created a set of

149 PT pseudo-transcripts by substituting the identified sequence variants into the B73 reference

150 transcripts. A single fasta file was created that contained two sequences per locus, one B73

151 transcript and one PT pseudo-transcript, and $\mathrm{B} 73 \mathrm{xPT}_{1}$ reads were re-aligned to this $\mathrm{F}_{1}$ pseudo-

152 reference using bowtie2 (Langmead \& Salzberg, 2012) with eXpress (Roberts et al., 2011);

153 (Roberts \& Pachter, 2013) recommended parameters. The number of reads per B73 and/or PT

154 transcript was then quantified using eXpress. A total of 9256 transcripts were identified to

155 contain polymorphisms, allowing estimation of ASE. Genes were considered to show ASE when

156 the number of associated reads assigned to B73 or PT transcripts was significantly different $\left(\chi^{2}\right.$

157 test against an equal number of counts; $\mathrm{p}<0.05$; Bonferroni correction for multiple tests) and the

158 absolute $\log 2$-transformed ratio of $\mathrm{PT} / \mathrm{B} 73$ reads was $>1$.

159

160 Gene Ontology annotation, enrichment analyses and comparison of ASE genes to published

161 data

162 Candidate ASE genes were assigned to Gene Ontology categories (release 52 available at

$163 \mathrm{ftp}: /$ ftp.gramene.org/pub/gramene). Obsolete annotations were replaced by the corresponding

164 "consider" or "replaced_by" category(ies) in the ontology file (go.obo) available at

165 http://www.geneontology.org/ (dated 2016-09-19). Categories associated with at least 10 genes

166 were considered in further analysis. Enrichment analyses were performed comparing ASE 
167 candidates against the 9256 polymorphic gene set, using the Bingo (Maere, Heymans \& Kuiper,

168 2005) Cytoscape (Shannon et al., 2003) plugin, using a hypergeometric test and controlling for

169 multiple tests using Benjamini and Hochberg False Discovery Rate at 1\%. Categories were called

170 PT up or PT down using a threshold of abs(median $\log \mathrm{FC})>=1$. Enrichment analysis of the 1938

171 TF targets gene set was performed against the 11299 genes represented on the microarray (see

172 "Reconstruction of gene co-expression network" section below).

173 Candidate ASE genes were cross-referenced to a published study describing

174 transcriptional responses in maize seedlings exposed to cold, heat, salt and UV stresses

175 (Makarevitch et al., 2015). Although a number of inbred lines were analyzed in the Makarevitch

176 study, only the B73 data was used in the comparison with the B73xPT transcriptome. Genes were

177 considered to show prior stress response (PSR) with respect to a given stress when: 1) identified

178 as ASE; 2) responding significantly to stress in the Makarevitch study (absolute $\log _{2}$ fold change

$179>1$; called as significant in the Makarevitch study; calls "up" or "on" in the published study were

180 considered here as "up", similarly, “down" or "off” were considered as “down”); 3) the sign of

181 ASE was concordant with the sign of stress response.

182 Fst values for population level differentiation between Mesoamerican and South

183 American highland and lowland maize populations (Takuno et al., 2015) were obtained from

184 https:/github.com/rossibarra/hilo paper/tree/master/fst; where multiple SNPs are associated with

185 a single gene, the values reported correspond to the SNP showing the highest Fst in

186 Mesoamerica.

187

188 Reconstruction of gene co-expression network

189 Publicly available maize Affymetrix microarray data was downloaded from the ArrayExpress 
190 website (http://www.ebi.ac.uk/arrayexpress/; experiments E-GEOD-10023, E-GEOD-12770, E-

191 GEOD-12892, E-GEOD-18846, E-GEOD-19785, E-GEOD-22479, E-GEOD-28479, E-GEOD-

192 31188, E-GEOD-40052, E-GEOD-41956, E-GEOD-48406, E-GEOD-48536, E-GEOD-54310,

193 E-GEOD-59533, E-GEOD-69659, E-MEXP-1222, E-MEXP-1464, E-MEXP-1465, E-MEXP-

194 2364, E-MEXP-2366, E-MEXP-2367, E-MEXP-3992). Low quality CEL files identified using

195 the arrayQualityMetrics (Kauffmann, Gentleman \& Huber, 2009) R package were discarded.

196 Using the sample data relationship file (sdrf) associated with each experiment, samples for B73

197 leaves were selected, resulting in a high quality, homogeneous dataset of 165 CEL files.

198 Probeset sequences for the maize Affymetrix microarray were aligned using seqmap (Jiang \&

199 Wong, 2008) to the AGPv3.22 transcripts with no mismatches allowed, and probesets whose

200 probe sequences did not align or aligned to transcripts corresponding to more than one locus

201 were discarded. Probesets that were represented by less than 4 probe sequences were also

202 discarded. This resulted in a list of 11299 probesets that unambiguously matched one locus. The

203 list of 11299 probesets was used to create a custom chip definition file (CDF) using the

204 ArrayInitiative python package (http://wellerlab.uncc.edu/ArrayInitiative/), and to filter the

205 original Affymetrix Maize.probe_tab file to create a custom probe_tab file. The custom CDF and

206 custom probe_tab file were then used to create the corresponding cdf and probe_tab R packages

207 using the makecdfenv (Irizarry et al., 2006) and AnnotationForge (Carlson and Pages, 2017) R

208 packages, respectively. The microarray name in the 165 CEL files was then modified to match

209 the custom cdf and probe_tab packages name, and these modified CEL files were normalized

210 using gcrma (Wu and Gentry, 2017). The resulting normalized dataset was then used as input for

211 the ARACNE algorithm (Margolin et al., 2006a); (Margolin et al., 2006b), and inference was

212 carried out for the 7 ASE and stress-responsive transcription factors (see Results) at DPI 0.1 as 
213 previously described (Chávez Montes et al., 2014).

214

215 RESULTS

216 A total of 2386 genes exhibited allele specific expression in the B73xPT $F_{1}$ hybrid

217 To identify regulatory variation associated with stress-related genes, high throughput sequencing

218 was used to quantify transcript abundance in leaves harvested from an $F_{1}$ seedling generated

219 from the cross between the Mexican highland landrace PT and the reference line B73. Alignment

220 to the B73 reference gene models identified 9256 genes containing at least one sequence variant

221 that could be used to distinguish the products of B73 and PT alleles. For $2386(26 \%)$ of these

2229256 polymorphic transcripts, the number of reads corresponding to the B73 allele differed

223 significantly ( $\mathrm{p}<0.05$; Bonferroni correction for multiple tests) from the number of reads

224 corresponding to the PT allele with an absolute $\log _{2}$ fold change $>1$, and these genes were

225 considered to exhibit allele specific expression (ASE; Supplementary Data 2 [F1_counts]). For

$2261412(59 \%)$ of the ASE candidate genes, accumulation of the PT transcript was lower than that of

227 the B73 transcript ( $\log _{2}$ PT/B73 <-1; hereafter, "PT-down"), while for the remaining $974(41 \%)$

228 of the ASE candidates, the PT transcript was accumulated at higher levels $\left(\log _{2}\right.$ PT/B73 $>1$;

229 hereafter, "PT-up").

230 To obtain an overview of the ASE candidates, a Gene Ontology (GO) analysis was

231 performed. The set of 2386 ASE candidates was not enriched for any specific GO categories with

232 respect to the 9256 polymorphic gene set, but, nonetheless, many individual genes belonged to

233 biological processes categories related to stress responses, including responses to heat (GO:

234 0009408), cold (GO: 0009409) and salt (GO: 0009651) (Fig. 1). Overall, 52 biological process

235 categories were represented by at least 10 genes. Of these, 38 (73\%) were PT-down (based on the 
236 median $\log _{2}$ PT/B73 of the associated genes), and 11 (21\%) were PT-up, and the remaining 3

237 categories had a median $\log _{2}$ PT/B73 close to 0 (Supplementary Data 3 [ASE_loci_GO_P]). A

238 similar pattern was observed for molecular function categories: 57 categories were associated

239 with at least ten ASE genes, 42 PT-down, 12 PT-up and 3 showing no trend (Supplementary

240 Data 4 [ASE_loci_GO_F]).

242 A total of 277 genes showed prior stress responses

243 To identify evidence of prior stress response (PSR) in PT, the ASE gene set was compared with a

244 previous study reporting changes in the transcriptome of B73 seedlings exposed to cold, heat, salt

245 or UV treatments (Makarevitch et al., 2015). Of these treatments, cold and UV stress are directly

246 relevant to plant performance in the highland niche, and salt stress may be considered to some a

247 extent a proxy for drought conditions. PT is not predicted to be adapted to heat stress, and, as

248 such, the heat treatment provides an interesting contrast to the other conditions, although, as

249 described below, many genes in this study were responsive to multiple stresses. A total of 1407

250 stress responsive genes identified in the Makarevitch study were present also in the 9256

251 polymorphic gene set for which ASE had been evaluated (Supplementary Data 2 [F1_counts]).

252 Of these 1407 genes, 432 (31\%) showed ASE, a slight enrichment compared with the 2386

253 (26\%) ASE genes in the 9256 polymorphic gene set as a whole (ASE, Makarevitch: 432; ASE,

254 non-Makarevitch: 1963; non-ASE, Makarevitch: 984; non-ASE, non-Makarevitch: 5886; $\chi^{2}=$

255 15.7, d.f. $=1, \mathrm{p}<0.001)$. From this 432 gene set, a gene was considered to exhibit PSR in PT if

256 the sign of ASE was concordant with the sign of B73 stress response: i.e. PT-up and induced by

257 stress in B73, or PT-down and repressed by stress in B73. On this basis, a set of 277 PSR

258 candidates was identified (Fig. 2A-D; Supplementary Data 5 [Maka_can_annot]). The majority 
259 of these 277 genes respond to two or more stress treatments (Fig.3A, C), but often in different

260 directions such that most present stress-specific PSR (Fig. 3B, C): 194 were identified as

261 showing PSR with respect to one treatment, 62 with respect to two, 17 with respect to three, and

2624 with respect to all four (Fig. 3C). Of the 277 genes, 92 showed PSR with respect to cold, 65

263 with respect to heat, 136 with respect to salt, and 92 with respect to UV (Fig. 3B). The number of

264 PSR genes with respect to any given stress was proportional to the number of genes responding

265 to that stress in the 1407 polymorphic gene set (cold, PSR: 92, non-PSR: 631; heat, PSR: 65,

266 non-PSR: 374; salt, PSR: 136, non-PSR: 736; UV, PSR: 92, non-PSR: 444; $\chi^{2}=5.2$, d.f. $=3, \mathrm{p}=$

267 0.16), and there was no indication of an enrichment for PSR with respect to any one of the four

268 treatments. In contrast to the complete ASE gene set, the majority of the 277 PSR genes were

269 PT-up (181 PT-up, 96 PT-down; Supplementary Data 5 [Maka_can_annot]), although this

270 general trend was not observed when the UV treatment was considered alone, where the majority

271 of PSR genes were PT-down (34 PT-up, 58 PT-down; Fig. 2D).

272

273 Hormone related genes and transcription factors showed constitutive stress responses in PT

274 A primary aim of the analysis was the definition of a small number of candidate genes for future

275 functional analysis. For this purpose, the PSR candidate genes were cross-referenced with the

276 classical maize gene list, a curated set of 4908 well-annotated genes, many linked with existing

277 functional data (the "combined set" gene list was obtained from

278 www.maizegdb.org/gene center/gene and filtered for unique gene identifiers). Of the 277 PSR

279 candidate genes, 48 were present in the classical gene list (Fig. 4; Supplementary Data 5

280 [Maka_can_annot]), including 9 genes associated with hormone homeostasis (Table 1) and 12

281 transcription factors (TFs; Table 2; (Jin et al., 2017)) that were considered of special interest. The 
282277 PSR candidates were cross referenced with a published study of population level

283 differentiation between Mesoamerican and South American highland and lowland maize

284 (Takuno et al., 2015). Fst estimates and significance were reported for 183 of the PSR

285 candidates, 22 which showed significant differentiation $(\mathrm{p}<0.1)$ between highland and lowland

286 Mesoamerican populations, including the hormone associated gene Czogl (GRMZM2G168474;

287 Supplementary Data 5 [Maka_can_annot]). The number of PSR candidates showing significant

288 Fst was as expected based on the overlap with the 1407 polymorphic gene set (Fst reported for

2891032 of 1407 genes; PSR, Fst $<0.1: 22$; PSR, Fst $>=0.1: 161$; non-PSR, Fst $<0.1: 100$; non-

290 PSR, Fst $>=0.1: 749 ; \chi^{2}<1$, d.f. $\left.=1, \mathrm{p}=1\right)$. To gain insight into potential $\mathrm{TF}$ targets and their

291 role in stress responses, a gene co-expression network for the PSR TFs was generated using

292 available maize Affymetrix microarray data and the ARACNE algorithm. Seven of the 12 TFs

293 were unambiguously identified in the maize Affymetrix microarray probeset, and were co-

294 expressed with 1938 genes (Supplementary Data 6 [tfs_ASE_01_suppl]). Co-expressed genes

295 represent potential targets of TF action, and, as such, may not themselves exhibit ASE. Indeed, of

296 the 1938 genes associated with the 7 TFs, 1097 were present in the polymorphic gene set, but

297 only 239 showed ASE. A total of 344 of the 1938 co-expressed genes (17\%) were responsive to

298 one or more stress treatments in the Makarevitch dataset (Fig. 5). A GO analysis detected

299 enrichment in the 1938 gene co-expression set with respect to translation, photosynthesis and

300 non-mevalonate isoprenoid pathway categories (Supplementary Data 7 [Bingo_aracne]).

301

302 DISCUSSION

303 From a starting set of 9256 polymorphic genes, we identified 2386 genes presenting allele

304 specific expression (ASE) in seedling leaves of an $\mathrm{F}_{1} \mathrm{~B} 73 \mathrm{xPT}$ hybrid individual. Comparison of 
305 our ASE gene list with a published dataset reporting B73 stress responses (Makarevitch et al.,

306 2015) identified a subset of 277 (out of 432) constitutive stress response (PSR) candidate genes

307 exhibiting a bias in transcript accumulation between PT and B73 alleles that mirrored the B73

308 response to one or more stress treatments. We did not observe an enrichment in GO term

309 assignments in either our ASE gene set or our PSR gene set. Nonetheless, given that ASE is

310 assaying cis-acting variation, a small number of genes associated with a given GO term may

311 have biological significance. The ASE gene set showed a bias towards lower expression of the

312 PT allele, reflected in the observation that the median value of ASE for the majority of GO

313 categories associated with ASE genes was also negative. Contrary to this trend, the subset of 277

314 selected PSR candidates showed a bias towards higher expression of the PT allele (181 of 277

315 presented higher expression of the PT allele), also reflected in the 1407 polymorphic genes that

316 overlapped with the Makarevitch set.

317 The bulk of the PSR gene set (206 of 277) responded to two or more stresses in the

318 Makarevitch B73 data, although in the majority (194 of 277) of cases the PSR itself was with

319 respect to a single stress only (Fig. 3), indicating that in many cases the sign (up/down) of the

320 response in B73 differed between stresses ( Supplementary Data 5 [Maka_can_annot]). By

321 definition, a gene could not show PSR with respect to both of two different stresses if the B73

322 responses were opposing. There was no evidence that genes showing opposing stress responses

323 in B73 were less likely to show ASE, and consequently, PSR in PT -- indeed, such genes were

324 actually more represented in the 277 PSR gene set (156 of 277; 56\%) than in the 1407

325 polymorphic and stress-responsive gene set (511 of 1407; 36\%). As such, many ASE events may

326 appear contradictory with respect to any given stress, i.e. PT-up ASE in genes repressed by B73

327 under stress, or PT-down ASE in genes induced by B73, especially in the context of cold and UV 
328 treatments, against which PT is considered to be well adapted. The spatio-temporal dynamics of

329 stress responses, however, are complex (e.g. Secco et al., 2013), and the resolution of the present

330 analysis, based on single time points and tissues, is limited. For example, the previously

331 characterized salt associated HD-ZIP transcription factor $\mathrm{Hb54}$ (also named $\mathrm{ZmHdz} 10$,

332 GRMZM2G041127; (Zhao et al., 2011); (Zhao et al., 2014) showed PT-up ASE, but was

333 repressed by salt treatment in the Makarevitch dataset, and consequently not considered to show

334 PSR. In this case, however, an additional functional study reports $H b 54$ to indeed be induced by

335 salt treatment (Zhao et al., 2014), albeit at a different time point, and with a different treatment

336 than that applied in the Makarevitch study $(300 \mathrm{mM} \mathrm{NaCl}$ for $20 \mathrm{hrs}$ in Makarevitch et al.;

$337200 \mathrm{mM} \mathrm{NaCl}$ for 3-12hrs in Zhao et al.). The study of Zhao and colleagues reports also that

338 constitutive expression of Hb54 in Arabidopsis and rice increases ABA sensitivity and tolerance

339 to drought and salt stress. In light of these data, PT-up ASE of Hb54 may indeed have biological

340 relevance, reflected by the number and nature of associated co-expression candidates (Fig. 5). In

341 the absence of further characterization, it would be premature to discount the potential

342 phenotypic impact, or adaptive value, of other examples where ASE in PT is opposed to the B73

343 stress response reported in the Makarevitch data.

344 Previous studies have highlighted the importance of cis-acting regulatory variation in

345 driving diversity in plant stress responses (e.g. (Waters et al., 2017). The generation of novel

346 physiological strategies to confront stress conditions may be most efficient when a change in the

347 regulation of a single gene has multiple, coordinated downstream consequences. Mechanistically,

348 two functional categories of clear interest are hormones, systemic regulators of physiology at the

349 whole plant level, and transcription factors (TFs), with their capacity to impact multiple

350 downstream targets through a regulatory cascade. The 277 PSR gene list includes eight hormone- 
351 related genes (Table 1), including genes implicated in the metabolism of cytokinin (Czogl;

352 (Martin et al., 2001), jasmonate (ZmOpr7; (Yan et al., 2012) and ethylene (Acco31; (Gallie \&

353 Young, 2004); (Avila et al., 2016). Additional PSR candidates included Ks2

354 (GRMZM2G093526; ZmKSL5), a gene related to the ent-kaurene synthase required for

355 gibberellin biosynthesis, but more likely involved in the more specialized kauralexin A series

356 biosynthesis pathway (Fu et al., 2016), and Thi2 (GRMZM2G074097), encoding a thiamine

357 thiazole synthase activity required for synthesis of the thiazole moiety during the production of

358 thiamin (vitamin $\mathrm{B}_{1}$; (Woodward et al., 2010). With regard to the latter candidate, B vitamins,

359 although not strictly plant hormones, can play an analogous role in whole plant physiology in the

360 face of stress (Hanson et al., 2016). Thiamin application has been reported to alleviate the impact

361 of abiotic stress in a number of crops, including maize (e.g. (Kaya et al., 2015), and thiamin

362 synthesis has been proposed as a target for transgenic biofortification (e.g. (Dong, Stockwell \&

363 Goyer, 2015). Identification of PT-up ASE associated with Thi2 represents a compelling target

364 for further analysis. Interestingly, both Thi2 and the related gene Thil (GRMZM2G018375) were

365 also co-expressed with the PT-up ASE drought and salt associated HD-ZIP TF Hb54 ((Zhao et

366 al., 2014); Table 2; Fig. 5; Supplementary Data 6). Interestingly, the PSR candidates Czog1 and

367 Ks2 were reported previously to show significant population level differentiation between

368 highland and lowland mesoamerican maize populations $\left(F_{s t} ; p=0.004, p=0.04\right.$, respectively;

369 (Takuno et al., 2015), indicating that variation at these loci may indeed play a role in local 370 adaptation.

371 In total, twelve TFs were present in the 277 PSR candidate gene set (Table 1), including

372 four NAC TFs. The NAC TFs are a plant-specific family implicated broadly in abiotic stress

373 responses (Nakashima et al., 2012); (Puranik et al., 2012); (Nuruzzaman, Sharoni \& Kikuchi, 
374 2013); (Nakashima, Yamaguchi-Shinozaki \& Shinozaki, 2014), previously proposed as a target

375 for engineering multiple stress tolerance (Shao, Wang \& Tang, 2015). The potential role of NAC

376 TFs in a generalized stress response is reflected by the observation that the candidates

377 ZmNacTF5 (GRMZM2G162739), ZmNacTF25 (also named ZmNac111, GRMZM2G127379;

378 (Mao et al., 2015), ZmNacTF61 (GRMZM2G003715) and ZmNacTF70 (GRMZM2G312201)

379 responded to 3, 3, 2 and 1 stress treatments, respectively (Table 2). The genes ZmNacTF5 and 380 ZmNacTF25 showed PT-down ASE, and PSR with respect to salt and cold, respectively, while

381 the genes ZmNacTF61 and ZmNacTF70 showed PT-up ASE and PSR with respect to cold and

382 UV, respectively. In B73, insertion of a miniature inverted-repeat transposable element (MITE)

383 in the ZmNacTF25 promoter has been reported previously to be associated with reduced gene

384 expression (relative to a number of tropical lines) and increased susceptibility to drought (Mao et

385 al., 2015). The accumulation of ZmNacTF25 transcripts in B73, however, is reduced under cold

386 in the Makarevitch dataset, indicating a potential trade-off between temperate and tropical lines,

387 and possible relevance of the PT-down ASE in the highland niche. The gene ZmNacTF61 was

388 notable for strong PT-up ASE $\left(\log _{2}\right.$ PT/B73 $=3.26$ and 2.15), up-regulation under both cold and

389 UV stress, and association with a large number (116) of strongly cold- and UV- induced co-

390 expression candidates, including the jasmonate biosynthetic genes $\operatorname{Opr} 7$ and $\operatorname{Lox} 4$ (Fig. 4; Fig. 5;

391 Supplementary Data 6).

392 Candidate PSR genes presented here were identified on the basis of ASE under benign

393 conditions. Investigation of the degree to which ASE is maintained under stress conditions is

394 required to determine whether the level of expression of these candidates remains plastic in PT,

395 albeit with an expression level different from B73, or whether expression has been canalized to a

396 constitutively responsive state (Waddington, 1942); (Levins, 1968); (von Heckel, Stephan \& 
397 Hutter, 2016). Nonetheless, the potential to identify relevant cis-regulatory variation through

398 exploration of the transcriptome under benign conditions presents an attractive avenue to

399 investigate stress response and local adaptation. A number of the candidates identified here

400 suggest testable predictions regarding hormone accumulation and expression of candidate TF

401 targets in the PT landrace. In a number of cases, ASE was observed in genes reported previously

402 to show significant genetic differentiation between lowland and highland Mexican maize

403 populations, offering further evidence of a link to adaptation to the highland niche

404 (Supplementary Data 5 [Maka_can_annot]). A recent study in monkey flower (Mimulus guttatus)

405 using ASE analysis to compare locally adapted coastal and inland accessions has found cis-

406 regulatory effects to be the main driver for regulatory variation, providing a precedent for the

407 approach proposed here (Gould, Chen \& Lowry, 2017). Validation of specific candidate genes

408 will require functional characterization, but it is anticipated that this will be greatly facilitated by

409 continued development of resources for maize reverse genetics and the generation of

410 introgression lines derived from Mexican highland maize.

411

\section{CONCLUSIONS}

413 Expression differences were observed between PT and B73 alleles under benign conditions that

414 mirror the B73 response to cold, heat, salt and/or UV treatments. The observed patterns of

415 expression indicate the presence of cis-acting regulatory variation differentiating the PT landrace

416 from the B73 reference inbred. Regulatory variants linked to classical genes associated with

417 signaling and stress-responses potentially contribute to the adaptation of PT to the Mexican

418 highland environment.

419

420 ACKNOWLEDGEMENTS 
421 We acknowledge Patrice Dubois for assistance in the generation of $F_{1}$ seed stock, and Patrick

422 Schnable and Cheng Ting Yeh for generation of transcriptome data.

423 
Table 1. ASE and stress-responsive hormone-related genes. List of genes involved in hormone biosynthesis, transport or catabolism present in the 277 PSR gene set. ASE call indicates biased expression of the PT allele (1) or B73 allele (-1). Response to stress indicates the name of the stress for which the gene was called as differentially expressed in the Makarevitch dataset. Constitutive response indicates the stress condition for which the sign of the ASE call and the stress response coincide.

\begin{tabular}{|c|c|c|c|c|c|c|}
\hline Gene id & Symbo & $\begin{array}{l}\text { Molecular } \\
\text { function }\end{array}$ & Hormone & $\begin{array}{l}\text { AS } \\
\text { E } \\
\text { call }\end{array}$ & $\begin{array}{l}\text { Respons } \\
\text { e to } \\
\text { stress }\end{array}$ & $\begin{array}{l}\text { Constitutiv } \\
\text { e response }\end{array}$ \\
\hline $\begin{array}{l}\text { GRMZM2G07056 } \\
3\end{array}$ & -- & auxin efflux carrier & $\begin{array}{l}\text { auxin } \\
\text { transport }\end{array}$ & 1 & $\begin{array}{l}\text { heat, salt, } \\
\text { uv }\end{array}$ & heat, salt \\
\hline $\begin{array}{l}\text { GRMZM2G07263 } \\
2\end{array}$ & --- & auxin efflux carrier & $\begin{array}{l}\text { auxin } \\
\text { transport }\end{array}$ & 1 & $\begin{array}{l}\text { heat, salt, } \\
\text { uv }\end{array}$ & heat, salt \\
\hline $\begin{array}{l}\text { GRMZM2G11259 } \\
8\end{array}$ & -- & auxin efflux carrier & $\begin{array}{l}\text { auxin } \\
\text { transport }\end{array}$ & 1 & $\begin{array}{l}\text { heat, salt, } \\
\text { uv }\end{array}$ & heat, salt \\
\hline $\begin{array}{l}\text { GRMZM2G47514 } \\
8\end{array}$ & -- & auxin efflux carrier & $\begin{array}{l}\text { auxin } \\
\text { transport }\end{array}$ & 1 & heat, salt & heat, salt \\
\hline $\begin{array}{l}\text { GRMZM2G07252 } \\
9\end{array}$ & Acco31 & $\begin{array}{l}1- \\
\text { aminocyclopropane } \\
\text {-1-carboxylate } \\
\text { oxidase }\end{array}$ & $\begin{array}{l}\text { ethylene } \\
\text { biosynthesis }\end{array}$ & 1 & $\begin{array}{l}\text { cold, } \\
\text { heat, salt, } \\
\text { uv }\end{array}$ & $\begin{array}{l}\text { cold, heat, } \\
\text { salt, uv }\end{array}$ \\
\hline $\begin{array}{l}\text { GRMZM2G02076 } \\
1\end{array}$ & -- & $\begin{array}{l}\text { putative } \\
\text { cytochrome P450 }\end{array}$ & $\begin{array}{l}\text { brassinosteroi } \\
\text { d catabolism }\end{array}$ & -1 & $\begin{array}{l}\text { cold, salt, } \\
\text { uv }\end{array}$ & cold, uv \\
\hline
\end{tabular}




\begin{tabular}{|l|l|l|l|l|l|l|}
\hline & & (castasterone C-26 & & & & \\
\hline 1 & hydroxylase) & & & & \\
\hline GRMZM2G14828 & Opr7 & 12 -oxo- & jasmonate & -1 & salt, uv & salt \\
\hline GRMZM2G16847 & Czog1 & cis-zeatin O- & cytokinin & 1 & salt & salt \\
\hline & & glucosyl transferase & homeostasis & & & \\
\hline
\end{tabular}


Table 2. ASE and stress-responsive TFs. List of TFs present in the 277 PSR gene set. PlantTFDB family indicates the TF family according to the PlantTFDB (Jin et al. 2017). ASE call indicates biased expression of the PT allele (1) or B73 allele (-1). Response to stress indicates the name of the stress for which the gene was called as differentially expressed in the Makarevitch dataset. PSR indicates the stress condition for which the sign of the ASE call and the stress response coincide. In Affymetrix array indicates of the TF is represented in the maize Affymetrix microarray.

\begin{tabular}{|c|c|c|c|c|c|c|}
\hline Gene id & Symbol & $\begin{array}{l}\text { PlantTFDB } \\
\text { family }\end{array}$ & $\begin{array}{l}\text { ASE } \\
\text { call }\end{array}$ & $\begin{array}{l}\text { Response } \\
\text { to stress }\end{array}$ & PSR & $\begin{array}{l}\text { In } \\
\text { Affymetrix } \\
\text { array? }\end{array}$ \\
\hline GRMZM2G159937 & Bhlh57 & bHLH & 1 & $\begin{array}{l}\text { cold, salt, } \\
\text { uv }\end{array}$ & uv & no \\
\hline GRMZM2G148333 & Ereb202 & ERF & 1 & uv & uv & yes \\
\hline GRMZM2G010920 & Glk18 & G2-like & -1 & heat, uv & uv & no \\
\hline GRMZM2G127537 & $H b 11$ & HD-ZIP & 1 & salt, uv & salt & yes \\
\hline GRMZM2G041127 & Hb54/ZmHdz10 & HD-ZIP & 1 & $\begin{array}{l}\text { cold, heat, } \\
\text { salt }\end{array}$ & cold & yes \\
\hline GRMZM2G049695 & Mybr24 & $\begin{array}{l}\text { MYB- } \\
\text { related }\end{array}$ & 1 & salt, uv & $\begin{array}{l}\text { salt, } \\
\text { uv }\end{array}$ & no \\
\hline GRMZM2G121753 & Mybr89 & $\begin{array}{l}\text { MYB- } \\
\text { related }\end{array}$ & -1 & $\begin{array}{l}\text { cold, salt, } \\
\text { uv }\end{array}$ & uv & no \\
\hline GRMZM2G127379 & NacTF25/ZmNAC111 & NAC & -1 & $\begin{array}{l}\text { cold, salt, } \\
\text { uv }\end{array}$ & cold & no \\
\hline
\end{tabular}




\begin{tabular}{|c|c|c|c|c|c|c|}
\hline GRMZM2G162739 & NacTF5 & NAC & -1 & $\begin{array}{l}\text { cold, salt, } \\
\text { uv }\end{array}$ & salt & yes \\
\hline GRMZM2G003715 & NacTF61 & NAC & 1 & cold, uv & $\begin{array}{l}\text { cold, } \\
\text { uv }\end{array}$ & yes \\
\hline GRMZM2G312201 & NacTF70 & NAC & 1 & uv & uv & yes \\
\hline GRMZM2G071907 & Wrky50 & WRKY & 1 & salt & salt & yes \\
\hline
\end{tabular}


Figure 1. ASE candidate genes are assigned to a range of biological process Gene Ontology categories. Hierarchical tree of Gene Ontology biological process categories represented in ASE loci. Nodes represent categories, with the root GO:0008150 biological process as the uppermost node. Edges represent the parent-child (i.e. "is_a") relationship between categories. Node color indicates the median ASE ( $\log _{2}$ PT/B73) for the genes in the category, with light blue indicating negative values and dark red indicating positive values. Node size is proportional to the number of loci assigned to corresponding category. Some category names were abbreviated for clarity.

\section{[2 COLUMNS]}

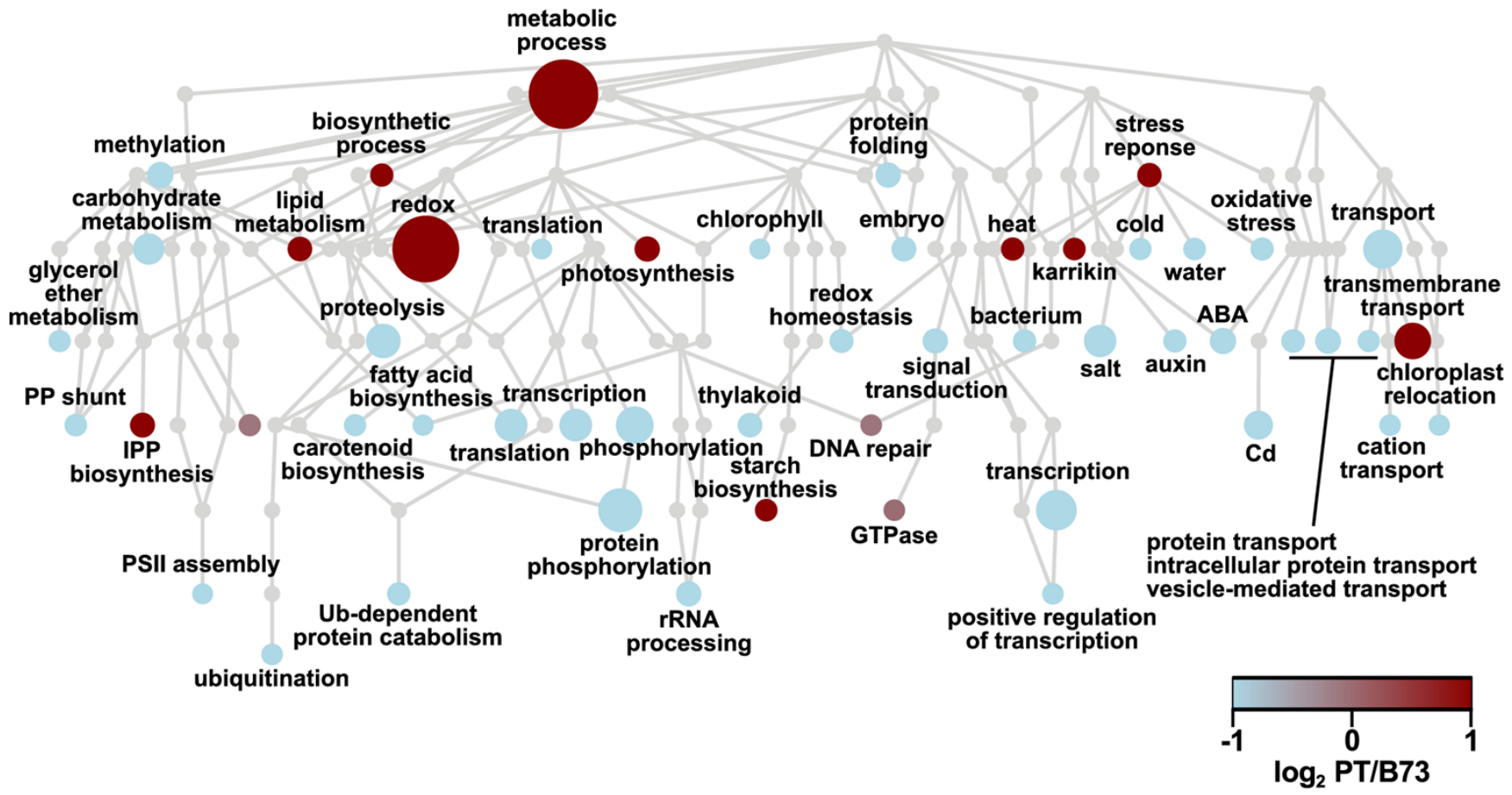


Figure 2. ASE identifies PSR in PT with respect to B73. ASE $\left(\log _{2} \mathrm{PT} / \mathrm{B} 73\right)$ in control $\mathrm{F}_{1}$ leaves for the 1407 sequence variant, stress-responsive gene set against B73 stress response $\left(\log _{2}\right.$ stress/control) for A) cold, B) heat, C) salt and D) UV treatments as reported in the Makarevitch dataset. Numbers in each quadrant represent the count of genes called as significant in ASE and stress comparisons. In each plot, the quadrants represent (clockwise from upper left) genes up ASE / down stress, up ASE / up stress, down ASE / up stress, down ASE / down stress. Genes called as up ASE / up stress or down ASE / down stress are considered to show PSR and are shown as filled circles. Other genes are shown as points. Axes through the origin are shown as red dashed lines. A number of genes outside the axis range are not shown, but are considered in the gene count.

\section{[TWO COLUMNS]}

A

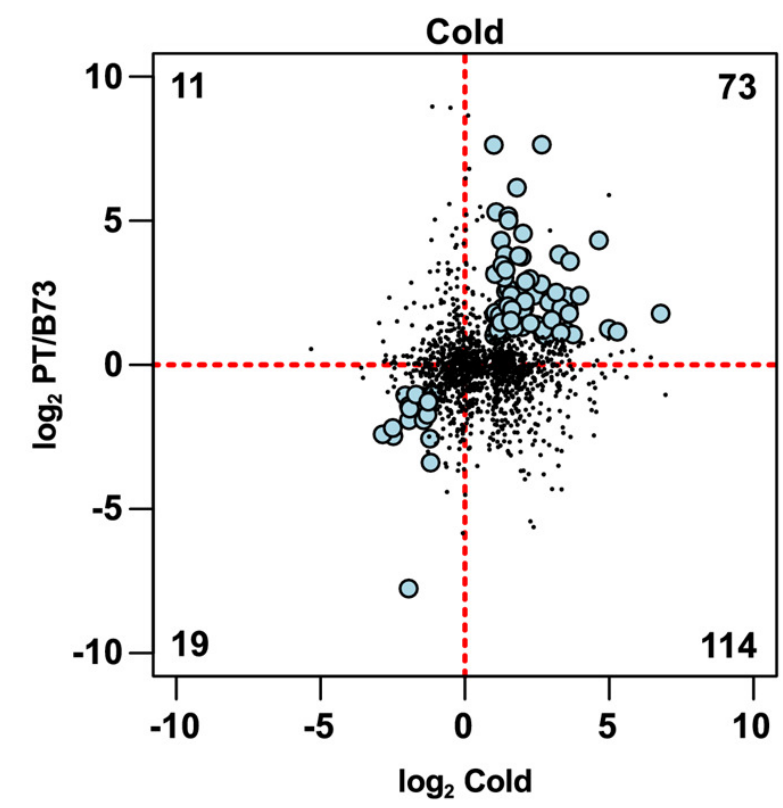

C

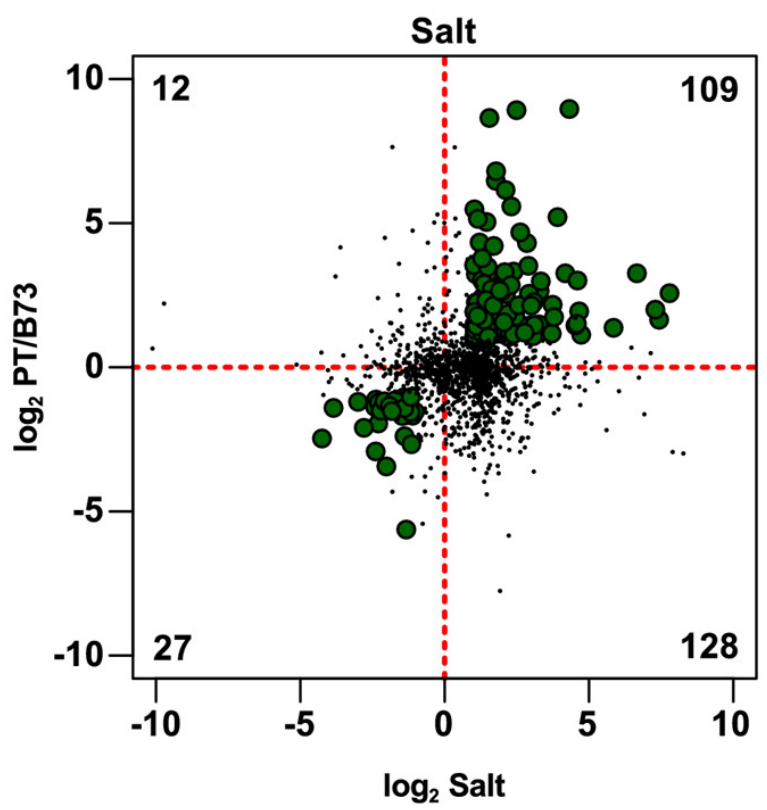

B

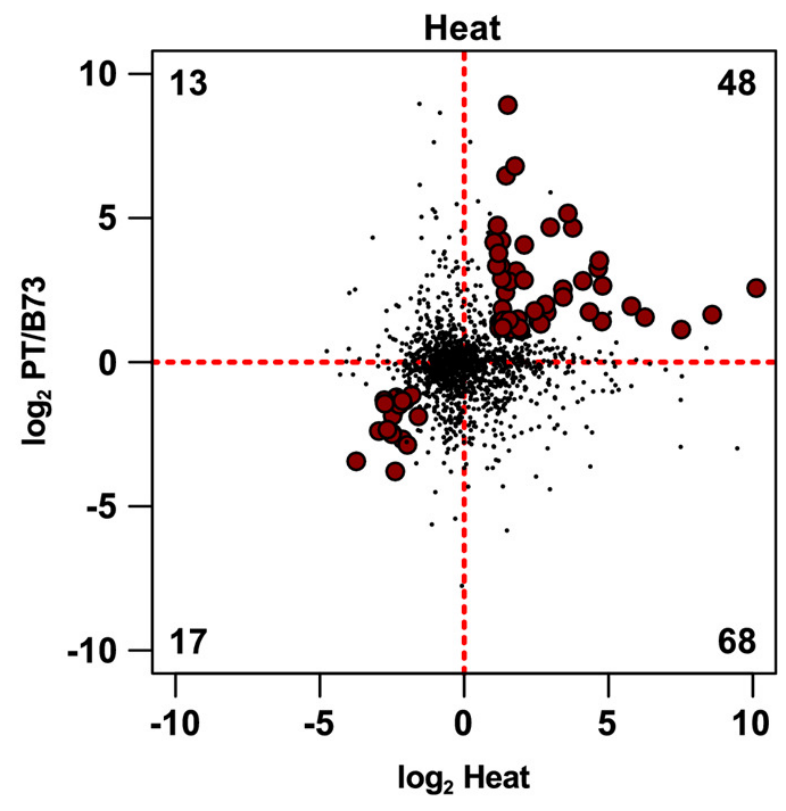

D

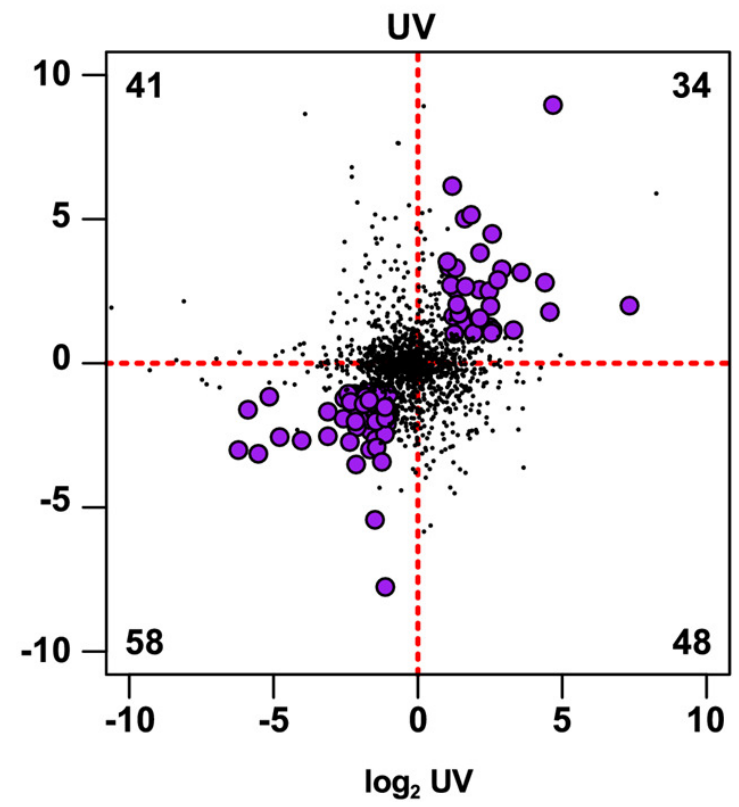


Figure 3. PSR candidates may respond to multiple stresses in B73. A) Number of genes from the 277 PSR gene set that responded to cold, heat, salt, UV or a combination of stresses in the Makarevitch B73 study. B) Number of genes called as PSR in PT with respect to each stress from the same 277 gene set. C) Counts with respect to number of stresses of genes in A and B. Numbers above bars give counts.

[ONE COLUMN]

\section{A}

B73 stress reponse

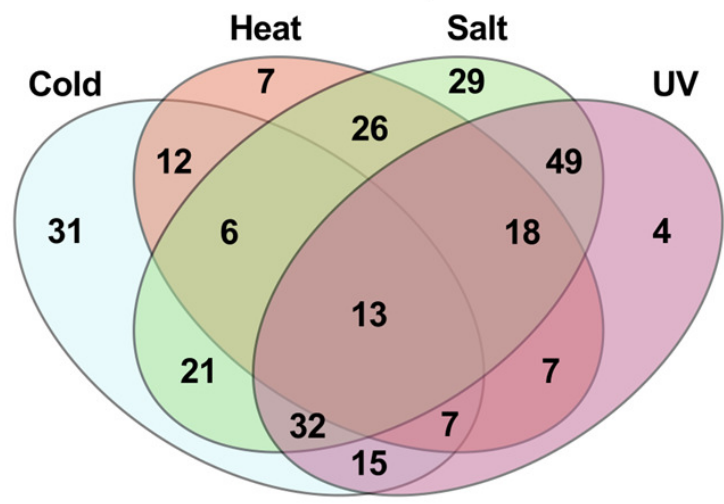

B
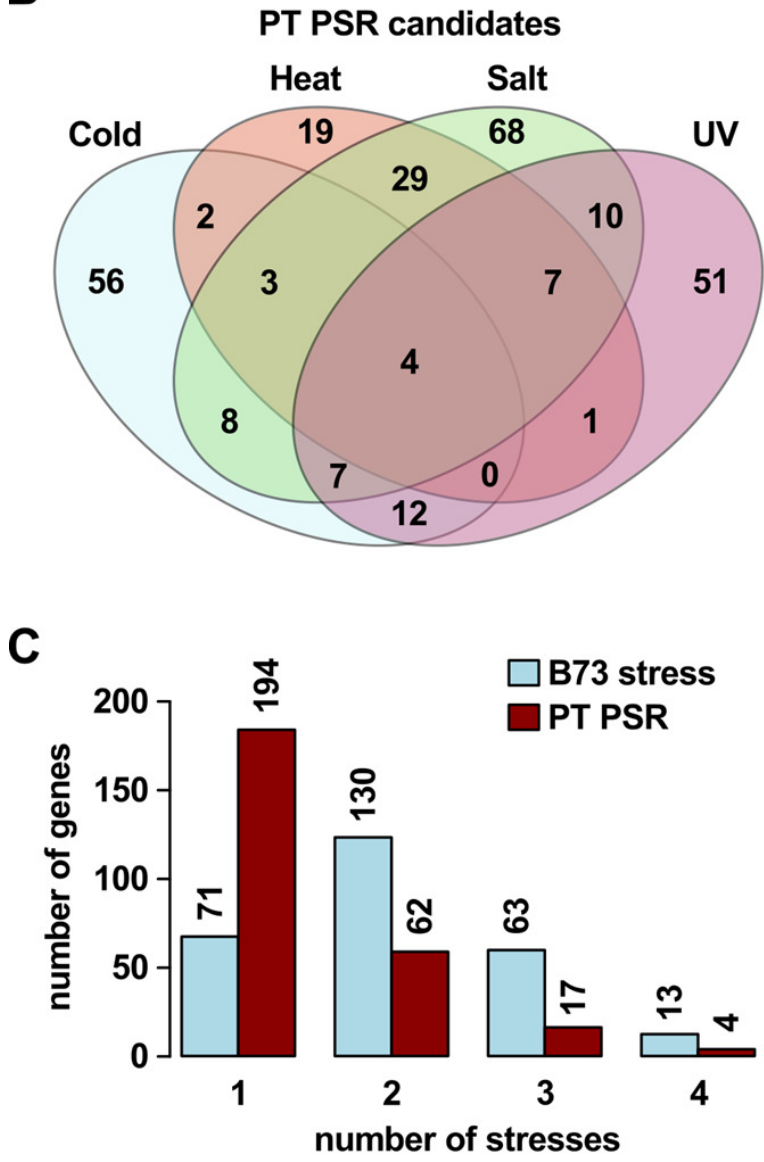
Figure 4. Classical PSR candidate genes. Heatmap representation of ASE ( $\log _{2}$ PT/B73) and B73 response to cold, heat, salt and UV stress $\left(\log _{2}\right.$ stress/control) as reported in the Makarevitch dataset for PSR candidates in the maize classical gene list. Asterisks (*) in the stress columns indicate a given gene was called as PSR with respect to that stress.

[ONE COLUMN]
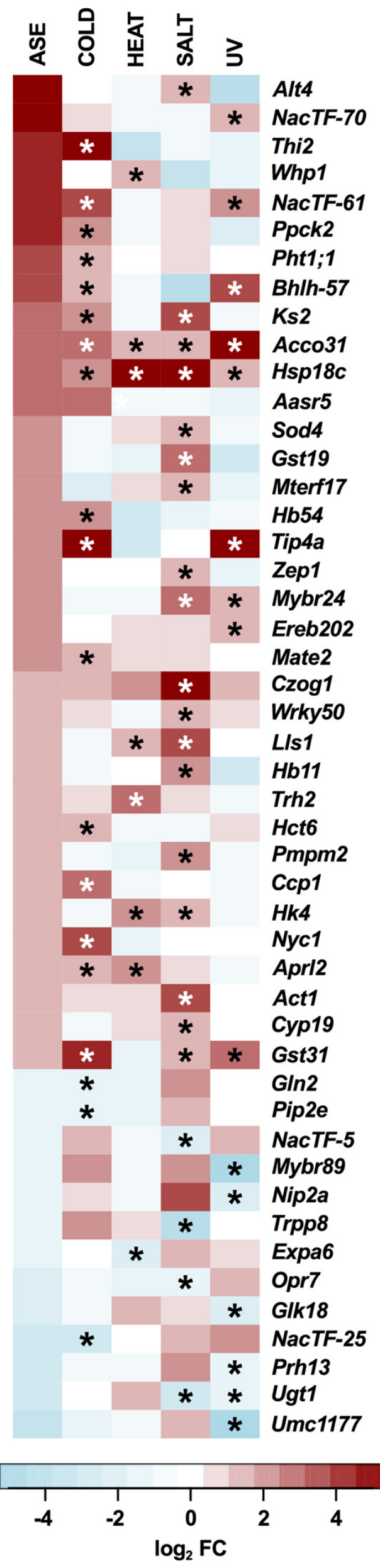
Figure 5. Co-expression networks for PSR TFs and their putative stress-responsive targets. Nodes represent genes and edges represent co-expression as calculated by the ARACNE algorithm at DPI 0.1. The central legend presents a network of seven PSR TFs (labeled centres of circles) with their co-expressed, stress-responsive (genes called up/on or down/off in the Makarevitch dataset) putative targets. Triangles indicate genes that were called as presenting ASE. The network was filtered to retain only co-expressed genes responsive A) cold, B) heat, C) salt or D) UV treatments. In the filtered networks the red and blue colors indicate up or down regulation (as $\log _{2} \mathrm{FC}$ from the Makarevitch dataset), respectively, under the corresponding stress.

[TWO COLUMNS] 

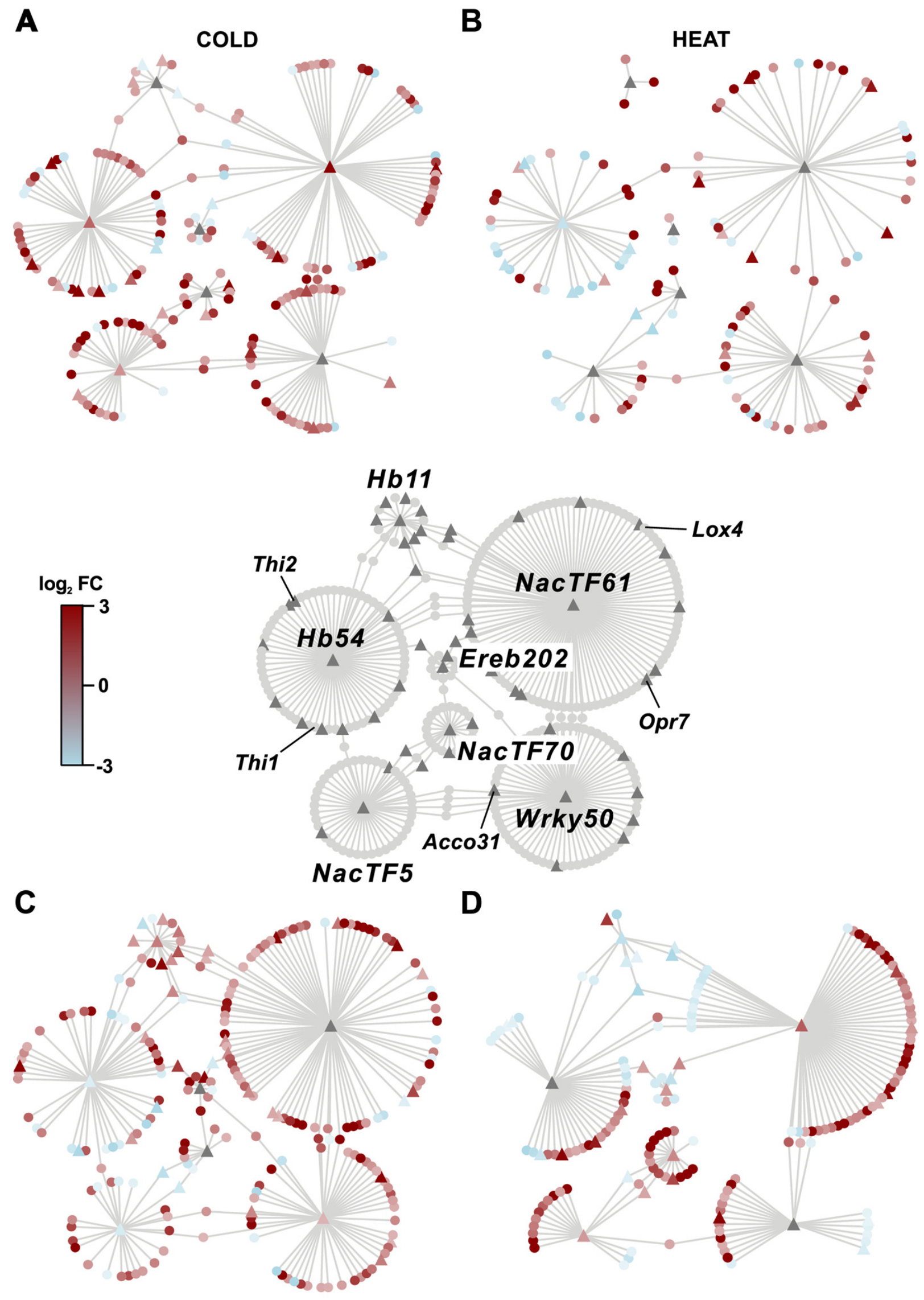

SALT

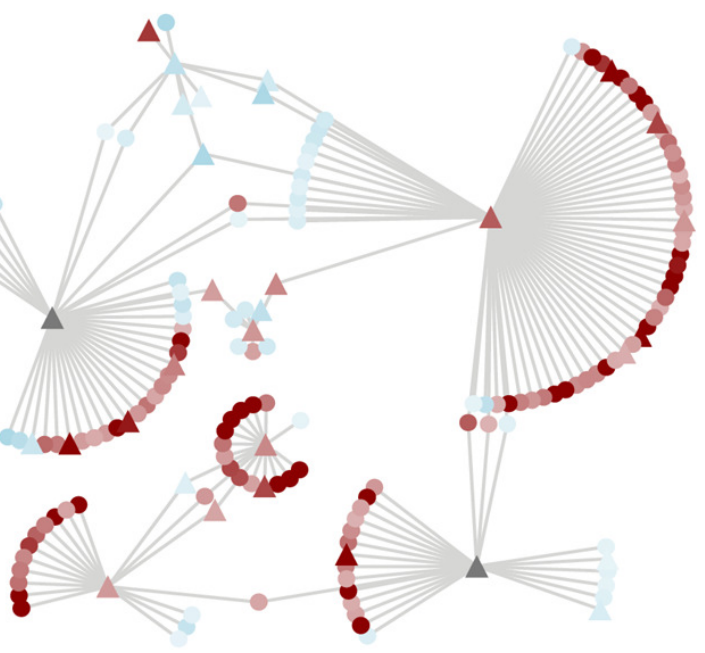

UV 


\section{REFERENCES}

Avila LM., Cerrudo D., Swanton C., Lukens L. 2016. Brevis plant1, a putative inositol polyphosphate 5-phosphatase, is required for internode elongation in maize. Journal of Experimental Botany 67:1577-1588.

Bolger AM., Lohse M., Usadel B. 2014. Trimmomatic: a flexible trimmer for Illumina sequence data. Bioinformatics 30:2114-2120.

Carlson M, Pages H. 2017. AnnotationForge: Code for Building Annotation Database Packages. R package version 1.18.0.

Chávez Montes RA., Coello G., González-Aguilera KL., Marsch-Martínez N., de Folter S., Alvarez-Buylla ER. 2014. ARACNe-based inference, using curated microarray data, of Arabidopsis thaliana root transcriptional regulatory networks. BMC Plant Biology 14:97.

Coop G., Witonsky D., Di Rienzo A., Pritchard JK. 2010. Using environmental correlations to identify loci underlying local adaptation. Genetics 185:1411-23.

Des Marais DL., Hernandez KM., Juenger TE. 2013. Genotype-by-Environment Interaction and Plasticity: Exploring Genomic Responses of Plants to the Abiotic Environment. Annual Review of Ecology, Evolution, and Systematics 44:5-29.

Dong W., Stockwell VO., Goyer A. 2015. Enhancement of Thiamin Content in Arabidopsis thaliana by Metabolic Engineering. Plant \& Cell Physiology 56:2285-2296.

Eagles HA., Lothrop JE. 1994. Highland Maize from Central Mexico?Its Origin, Characteristics, and Use in Breeding Programs. Crop science 34:11.

Fu J., Ren F., Lu X., Mao H., Xu M., Degenhardt J., Peters RJ., Wang Q. 2016. A Tandem Array of ent-Kaurene Synthases in Maize with Roles in Gibberellin and More Specialized Metabolism. Plant Physiology 170:742-751. 
Furbank RT., Tester M. 2011. Phenomics--technologies to relieve the phenotyping bottleneck. Trends in Plant Science 16:635-644.

Gallie DR., Young TE. 2004. The ethylene biosynthetic and perception machinery is differentially expressed during endosperm and embryo development in maize. Molecular Genetics and Genomics 271:267-281.

Gould BA., Chen Y., Lowry DB. 2017. The gene regulatory landscape of local adaptation. bioRxiv doi: 10.1101/145805.

Hannah MA., Wiese D., Freund S., Fiehn O., Heyer AG., Hincha DK. 2006. Natural genetic variation of freezing tolerance in Arabidopsis. Plant Physiology 142:98-112.

Hanson AD., Beaudoin GA., McCarty DR., Gregory JF. 2016. Does abiotic stress cause functional B vitamin deficiency in plants? Plant Physiology 172:2082-2097.

Hayano-Kanashiro C., Calderón-Vázquez C., Ibarra-Laclette E., Herrera-Estrella L., Simpson J. 2009. Analysis of gene expression and physiological responses in three Mexican maize landraces under drought stress and recovery irrigation. Plos One 4:e7531.

von Heckel K., Stephan W., Hutter S. 2016. Canalization of gene expression is a major signature of regulatory cold adaptation in temperate Drosophila melanogaster. BMC Genomics $17: 574$

Hilker M., Schwachtje J., Baier M., Balazadeh S., Bäurle I., Geiselhardt S., Hincha DK., Kunze R., Mueller-Roeber B., Rillig MC., Rolff J., Romeis T., Schmülling T., Steppuhn A., van Dongen J., Whitcomb SJ, Wurst S., Zuther E., Kopka J. 2016. Priming and memory of stress responses in organisms lacking a nervous system. Biological Reviews of the Cambridge Philosophical Society 91:1118-1133.

Houle D., Govindaraju DR., Omholt S. 2010. Phenomics: the next challenge. Nature Reviews. 
Genetics 11:855-866.

van Hulten M., Pelser M., van Loon LC., Pieterse CMLJ., Ton J. 2006. Costs and benefits of priming for defense in Arabidopsis. PNAS 103:5602-5607

Jiang C., Edmeades GO., Armstead I., Lafitte HR., Hayward MD., Hoisington D. 1999. Genetic analysis of adaptation differences between highland and lowland tropical maize using molecular markers. TAG Theoretical and Applied Genetics 99:1106-1119.

Jiang H., Wong WH. 2008. SeqMap: mapping massive amount of oligonucleotides to the genome. Bioinformatics 24:2395-2396.

Jin J., Tian F., Yang D-C., Meng Y-Q., Kong L., Luo J., Gao G. 2017. PlantTFDB 4.0: toward a central hub for transcription factors and regulatory interactions in plants. Nucleic Acids Research 45:D1040-D1045.

Kamthan A., Chaudhuri A., Kamthan M., Datta A. 2016. Genetically modified (GM) crops: milestones and new advances in crop improvement. TAG. Theoretical and Applied Genetics. Theoretische und Angewandte Genetik 129:1639-1655.

Kauffmann A., Gentleman R., Huber W. 2009. arrayQualityMetrics--a bioconductor package for quality assessment of microarray data. Bioinformatics 25:415-416.

Kaya C., Ashraf M., Sonmez O., Tuna AL., Polat T., Aydemir S. 2015. Exogenous application of thiamin promotes growth and antioxidative defense system at initial phases of development in salt-stressed plants of two maize cultivars differing in salinity tolerance. Acta physiologiae plantarum / Polish Academy of Sciences, Committee of Plant Physiology Genetics and Breeding 37.

Lafitte HR., Edmeades GO. 1997. Temperature effects on radiation use and biomass partitioning in diverse tropical maize cultivars. Field crops research 49:231-247. 
Langmead B., Salzberg SL. 2012. Fast gapped-read alignment with Bowtie 2. Nature Methods 9:357-359.

Lasky JR., Des Marais DL., Lowry DB., Povolotskaya I., McKay JK., Richards JH., Keitt TH., Juenger TE. 2014. Natural variation in abiotic stress responsive gene expression and local adaptation to climate in Arabidopsis thaliana. Molecular Biology and Evolution 31:22832296.

Lasky JR., Upadhyaya HD., Ramu P., Deshpande S., Hash CT., Bonnette J., Juenger TE., Hyma K., Acharya C., Mitchell SE., Buckler ES., Brenton Z., Kresovich S., Morris GP. 2015. Genome-environment associations in sorghum landraces predict adaptive traits. Science Advances 1(6):e1400218

Lemmon ZH., Bukowski R., Sun Q., Doebley JF. 2014. The role of cis regulatory evolution in maize domestication. PLoS Genetics 10:e1004745.

Levins R. Evolution in changing environments. Princeton, N.J. : Princeton University Press, 1968.: Princeton University Press.

Li H. 2011a. Improving SNP discovery by base alignment quality. Bioinformatics 27:11571158.

Li H. 2011b. A statistical framework for SNP calling, mutation discovery, association mapping and population genetical parameter estimation from sequencing data. Bioinformatics 27:2987-2993.

Li H. 2013. Aligning sequence reads, clone sequences and assembly contigs with BWA-MEM. arXiv:1303.3997.

Li H., Handsaker B., Wysoker A., Fennell T., Ruan J., Homer N., Marth G., Abecasis G., Durbin R., 1000 Genome Project Data Processing Subgroup. 2009. The Sequence Alignment/Map 
format and SAMtools. Bioinformatics 25:2078-2079.

Maere S., Heymans K., Kuiper M. 2005. BiNGO: a Cytoscape plugin to assess overrepresentation of gene ontology categories in biological networks. Bioinformatics $21: 3448-3449$.

Makarevitch I., Waters AJ., West PT., Stitzer M., Hirsch CN., Ross-Ibarra J., Springer NM. 2015. Transposable elements contribute to activation of maize genes in response to abiotic stress. PLoS Genetics 11:e1004915.

Mao H., Wang H., Liu S., Li Z., Yang X., Yan J., Li J., Tran L-SP., Qin F. 2015. A transposable element in a NAC gene is associated with drought tolerance in maize seedlings. Nature Communications 6:8326.

Margolin AA., Nemenman I., Basso K., Wiggins C., Stolovitzky G., Dalla Favera R., Califano A. 2006a. ARACNE: an algorithm for the reconstruction of gene regulatory networks in a mammalian cellular context. BMC Bioinformatics 7 Suppl 1:S7.

Margolin AA., Wang K., Lim WK., Kustagi M., Nemenman I., Califano A. 2006b. Reverse engineering cellular networks. Nature Protocols 1:662-671.

Martin RC., Mok MC., Habben JE., Mok DW. 2001. A maize cytokinin gene encoding an Oglucosyltransferase specific to cis-zeatin. Proceedings of the National Academy of Sciences of the United States of America 98:5922-5926.

Mercer K., Martínez-Vásquez Á., Perales HR. 2008. Asymmetrical local adaptation of maize landraces along an altitudinal gradient. Evolutionary applications 1:489-500.

Nakashima K., Takasaki H., Mizoi J., Shinozaki K., Yamaguchi-Shinozaki K. 2012. NAC transcription factors in plant abiotic stress responses. Biochimica et Biophysica Acta 1819:97-103. 
Nakashima K., Yamaguchi-Shinozaki K., Shinozaki K. 2014. The transcriptional regulatory network in the drought response and its crosstalk in abiotic stress responses including drought, cold, and heat. Frontiers in plant science 5:170.

Nuruzzaman M., Sharoni AM., Kikuchi S. 2013. Roles of NAC transcription factors in the regulation of biotic and abiotic stress responses in plants. Frontiers in microbiology 4:248.

Perales H., Golicher D. 2014. Mapping the diversity of maize races in Mexico. Plos One 9:e114657.

Prasanna BM. 2012. Diversity in global maize germplasm: characterization and utilization. Journal of Biosciences 37:843-855.

Puranik S., Sahu PP., Srivastava PS., Prasad M. 2012. NAC proteins: regulation and role in stress tolerance. Trends in Plant Science 17:369-381.

Rengel D., Arribat S., Maury P., Martin-Magniette M-L., Hourlier T., Laporte M., Varès D., Carrère S., Grieu P., Balzergue S., Gouzy J., Vincourt P., Langlade NB. 2012. A genephenotype network based on genetic variability for drought responses reveals key physiological processes in controlled and natural environments. Plos One 7:e45249.

Roberts A., Pachter L. 2013. Streaming fragment assignment for real-time analysis of sequencing experiments. Nature Methods 10:71-73.

Roberts A., Trapnell C., Donaghey J., Rinn JL., Pachter L. 2011. Improving RNA-Seq expression estimates by correcting for fragment bias. Genome Biology 12:R22.

Romero Navarro JA., Willcox M., Burgueño J., Romay C., Swarts K., Trachsel S., Preciado E., Terron A., Delgado HV., Vidal V., Ortega A., Banda AE., Montiel NO., Ortiz-Monasterio I., Vicente FS., Espinoza AG., Atlin G., Wenzl P., Hearne S., Buckler ES. 2017. A study of allelic diversity underlying flowering-time adaptation in maize landraces. Nature Genetics 
49:476-480.

Ruiz Corral JA., Durán Puga N., Sánchez González J de J., Ron Parra J., González Eguiarte DR., Holland JB., Medina García G. 2008. Climatic adaptation and ecological descriptors of 42 mexican maize races. Crop science 48:1502.

Secco D., Jabnoune M., Walker H., Shou H., Wu P., Poirier Y., Whelan J. 2013. Spatio-temporal transcript profiling of rice roots and shoots in response to phosphate starvation and recovery. The Plant Cell 25:4285-4304.

Shannon P., Markiel A., Ozier O., Baliga NS., Wang JT., Ramage D., Amin N., Schwikowski B., Ideker T. 2003. Cytoscape: a software environment for integrated models of biomolecular interaction networks. Genome Research 13:2498-2504.

Shao H., Wang H., Tang X. 2015. NAC transcription factors in plant multiple abiotic stress responses: progress and prospects. Frontiers in plant science 6:902.

Sood S., Flint-Garcia S., Willcox MC., Holland JB. 2014. Mining Natural Variation for Maize Improvement: Selection on Phenotypes and Genes. In: Tuberosa R, Graner A, Frison E eds. Genomics of Plant Genetic Resources. Dordrecht: Springer Netherlands, 615-649.

Springer NM., Stupar RM. 2007a. Allelic variation and heterosis in maize: how do two halves make more than a whole? Genome Research 17:264-275.

Springer NM., Stupar RM. 2007b. Allele-specific expression patterns reveal biases and embryospecific parent-of-origin effects in hybrid maize. The Plant Cell 19:2391-2402.

Swanson-Wagner R., Briskine R., Schaefer R., Hufford MB., Ross-Ibarra J., Myers CL., Tiffin P., Springer NM. 2012. Reshaping of the maize transcriptome by domestication. Proceedings of the National Academy of Sciences of the United States of America 109:11878-11883. 
Takuno S., Ralph P., Swarts K., Elshire RJ., Glaubitz JC., Buckler ES., Hufford MB., RossIbarra J. 2015. Independent molecular basis of convergent highland adaptation in maize. Genetics 200:1297-1312.

Waddington CH. 1942. Canalization of Development and the Inheritance of Acquired Characters. Nature 150:563-565.

Waters AJ., Makarevitch I., Noshay J., Burghardt LT., Hirsch CN., Hirsch CD., Springer NM. 2017. Natural variation for gene expression responses to abiotic stress in maize. The Plant Journal: for Cell and Molecular Biology 89:706-717.

Woodward JB., Abeydeera ND., Paul D., Phillips K., Rapala-Kozik M., Freeling M., Begley TP., Ealick SE., McSteen P., Scanlon MJ. 2010. A maize thiamine auxotroph is defective in shoot meristem maintenance. The Plant Cell 22:3305-3317.

Wu J., Gentry RI. 2017. gcrma: Background Adjustment Using Sequence Information. $R$ package version 2.48 .0 .

Yan Y., Christensen S., Isakeit T., Engelberth J., Meeley R., Hayward A., Emery RJN., Kolomiets MV. 2012. Disruption of OPR7 and OPR8 reveals the versatile functions of jasmonic acid in maize development and defense. The Plant Cell 24:1420-1436.

Zhang X., Borevitz JO. 2009. Global analysis of allele-specific expression in Arabidopsis thaliana. Genetics 182:943-954.

Zhao Y., Ma Q., Jin X., Peng X., Liu J., Deng L., Yan H., Sheng L., Jiang H., Cheng B. 2014. A novel maize homeodomain-leucine zipper (HD-Zip) I gene, Zmhdz10, positively regulates drought and salt tolerance in both rice and Arabidopsis. Plant \& Cell Physiology 55:11421156.

Zhao Y., Zhou Y., Jiang H., Li X., Gan D., Peng X., Zhu S., Cheng B. 2011. Systematic analysis 
of sequences and expression patterns of drought-responsive members of the HD-Zip gene family in maize. Plos One 6:e28488. 